\title{
Short-term Load Prediction of Power System Based on Genetic Neural Network
}

\author{
Shiwei $\mathrm{Li}^{1, \mathrm{a}}$, Yulong $\mathrm{Li}^{2, \mathrm{~b}}$ \\ ${ }^{1}$ School of electronic and information engineering, LanZhou JiaoTong University, Lanzhou, 730070, \\ China \\ ${ }^{2}$ Experiment center on computer science, LanZhou JiaoTong University, Lanzhou, 730070, China \\ aemail: lishiwei8899@126.com, bemail: liyulonglanzhou@126.com
}

Keywords: Genetic algorithm; Neural network; L-M algorithm;Power system; Load forecasting

\begin{abstract}
As the basic content of power system's operation management and real-time control, the short-term load forecasting is significant for power system to run safely and economically. A short-term power system load predicting model based on genetic neural network is proposed, which take many factors into account, such as temperature, holidays and so on. In order to solve the problems of long convergence time and being easy to fall into local minimum of BP neural network, genetic algorithm (GA) is used to make a global searching for the initial weights and thresholds to solve the problems of long convergence time of BP neural network, and the Levenberg-Marquardt (L-M) method is used to train the network quickly. Based on the historical actual data, the forecasting results by the proposed method is more precise than those by BP neural network model, providing an effective way to forecast short-term power system load.
\end{abstract}

\section{Introduction}

With unceasing improvement of power system's automation degree, and as a major content of power system's operation management and actual-time control, the security and economy to power system running by short-term load prediction of power system have significant meanings. Before the 1980s, the traditional random time series prediction is a chief prediction method, however, it is difficult to confirm statistic features needed in its modeling and identify an non-stable state. In 1970, Sijesen conducted the load prediction by means of spectral decomposition, which introduced the load prediction into the actual syste ${ }^{[1]} \mathrm{m}$. Since $1990 \mathrm{~s}$, the gray prediction model and artificial neutral network are used for load prediction and achieve an ideal effect ${ }^{[2]}$. In order to solve traditional neutral network, the BP training method, which exists problems like slow learning speed and easiness to get in local minimum, is used mostly. The paper adopts the neutral network training with L-M (Levenberg-Marquardt) algorithm, and meanwhile takes advantage of an improved genetic algorithm to optimize the initial weight and threshold and actualizes global optimization, thus improving prediction accuracy of power system load.

\section{Principle of short-term load prediction model}

The prediction of power system load belongs to time series prediction, and adoption of neutral network to do time series prediction attributes to an nonparametric prediction method, which has no need of accurate system model and has a more broader application range than parameter prediction method. The time series prediction can be understood to be mathematical mapping to compute future trends by historical records, and the mathematical model, which has $\mathrm{k}$ steps in advance to forecast future trends by historical data at point $\mathrm{m}$, is:

$$
\begin{aligned}
& X(i), X(i+1), \cdots, X(i+k) \\
& =p(X(i-1), X(i-2), X(i-m))
\end{aligned}
$$

Thereof: $i=m+1, m+2, \cdots, \frac{N-m-k}{s}$ stands for the number of learning sample; $\mathrm{N}$ is length of 
sample data; s means the number of sliding point (usually its value is 1). Because the L-M neutral network can be approximate to a mapping relation implied in sample data, the paper utilizes the L-M neutral network optimized by genetic algorithm to solve a problem of time series prediction.

\section{Genetic neutral network model}

\section{Levenberg-Marquardt algorithm}

The L-M (Levenberg-Marquardt) algorithm mixes the Gauss-Newton method and the gradient descent method. Because the L-M algorithm uses approximate second derivative information which is faster than the gradient descent method; meanwhile, the L-M algorithm has no need to do excess parameter adjustment and is widely applied in many occasion of parameter optimization ${ }^{[3]}$. When using the neutral network training with L-M algorithm, the weight adjustment formula is expressed as follows:

$$
\Delta W=\left(\boldsymbol{J}^{T} \boldsymbol{J}+\boldsymbol{\mu I}\right)^{-1} \boldsymbol{J}^{T} \boldsymbol{E}
$$

Thereof, $\Delta \boldsymbol{W}$ is a revised amount of neutral network weight; $\boldsymbol{E}$ is an output error of neutral network; $\boldsymbol{J}$ stands for the Jacobian matrix that the output error vesus the network weight differential; $\mu$ is a scalar, when $\mu=0$, the L-M algorithm is the exact Gauss-Newton method, and when the $\mu$ value is bigger, then it more closes to the gradient descent method. In actual application, the L-M algorithm has a second-order convergence speed and needs less times of iteration, and it is not only characterized by local convergence of Newton method, but also possesses an overall situation feature of gradient descent method. The practice shows that the L-M algorithm is capable of effectively solving deficiencies in traditional BP algorithm, shortening training time and improving training accuracy.

\section{Genetic neutral network algorithm}

The genetic algorithm belongs to a kind of random search and optimization algorithm established during a long-term evolution process by simulating genetic variance of biological population. It has group optimization and natural reinforcement learning capacity, making it have wholeness, parallelism, rapidity and adaptability and applying to optimize the structure weight system and learning rules of neutral network. The paper utilizes the genetic algorithm to optimize the initial weight and threshold of neutral network.

The paper, first, takes advantage of an overall-situation optimization strategy of genetic algorithm in order to search the initial weight and threshold of neutral network in whole situation and make them fall into accurate neighborhood of optimal solution in overall situation; second, uses the L-M algorithm to do second accurate training to the initial weight and threshold of neutral network, thus making them convergent to optimal solution in overall situation. The genetic neutral network adopted in the paper combines the overall situation convergence ability of genetic algorithm with the rapid convergence feature of L-M algorithm, which effectively solves a problem that the traditional BP neutral network has long training time and is easy to fall into local minimum and validly improves the learning effect of neutral network ${ }^{[4]}$, and the algorithm flowchart sees the figure 1:

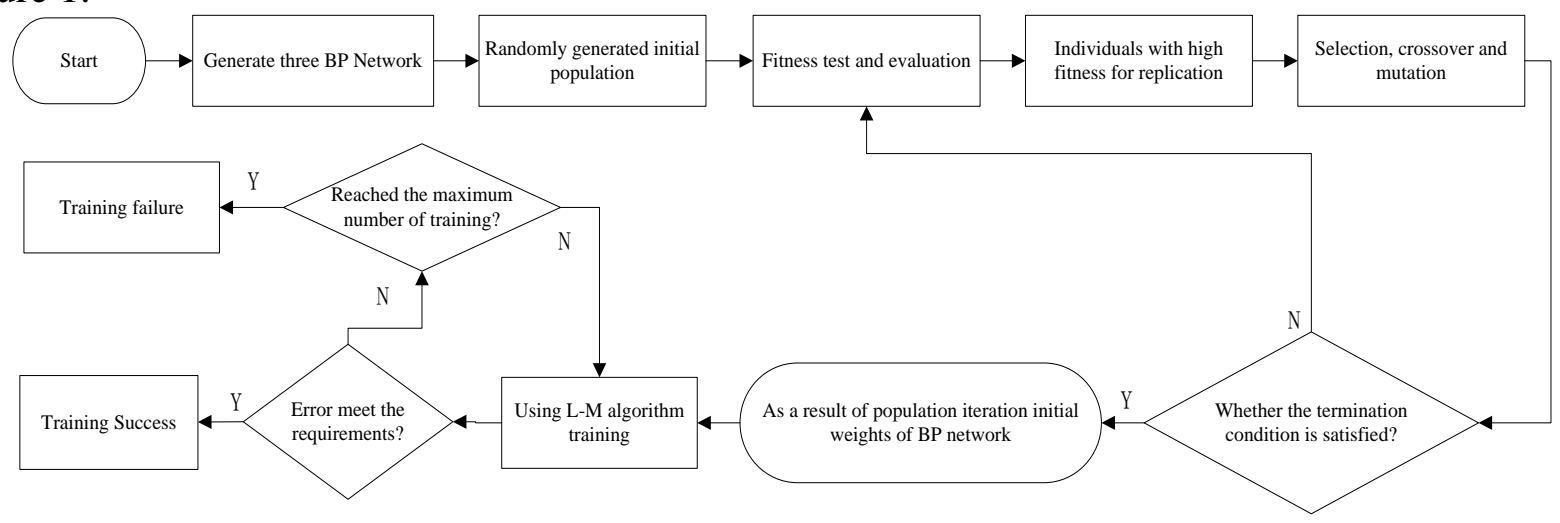

Fig.1. Flowchart of genetic neural network algorithm 


\section{Example analysis}

\section{Selection of genetic neutral network parameter}

Selection of neutral network parameter. According to the Kolmogorov theorem, a three-layer forward neutral network can approximate to any continuous nonlinear function, hence, the neutral network of prediction model of genetic neutral network load in the paper uses a three-layer structure, namely, one input layer, one hidden layer and one output layer ${ }^{[5]}$. The number of input layer's neuron is 27 , the 1 st to $24^{\text {th }}$ neuron node are input load values of power system of 24 discrete time points in 24 hours the day before, the $25^{\text {th }}$ to $26^{\text {th }}$ neuron nodes are input the highest and lowest temperature that very day, and the $27^{\text {th }}$ neuron node are input the impact factor of holiday; the number of hidden layer's neuron is set to be 27; the number of output layer's neuron is 24, and neurons are input load values of power system of 24 discrete time points in 24 hours that very day. The activation function of output layer is the purelin function, and the hidden layer has the tansig function as its activation function and the trainlm function as its network training function; the network learning speed rate is 0.01 and the training goal position is 0.00001 .

Selection of genetic algorithm parameter. The optimization process of genetic algorithm carries out the genetic operation in accordance with the size of fitness function, so the reciprocal of error square function selected by the paper is conducted as the algorithm fitness function; the gene length of neutral network weight individual is $P=27 \times 13+13 \times 24+13+24=700$, because the gene digit capacity is longer, so the paper utilizes a floating point coding way; the size of initial population is 50; a roulette selection mode is used; the arithmetic crossover probability is confirmed to be 0.9 ; the non-uniform mutation probability is 0.09 ; and the termination evolution algebra is 100.

\section{Simulation and result analysis of load prediction}

The load of power system is impacted by many factors such as air temperature, period of time, holiday and environment. The paper comprehensively takes account of many factors and adds impact factors which include air temperature, period of time and holiday into the neutral network input in order to eager to get a more precise prediction model. The paper mixes actual data of power system in a certain area of Deyang, Yunnan Province to test the load prediction effect of genetic neutral network. The week load statistic data in this area from 30, March, 2006 to 5, April, 2006 are used for testing. A day is divided into 24 periods of time, the load data of 6 days before this day are training samples of neutral network, the load data of 1 day before this day are input vectors of neutral network, and load data of the second day are goal vectors of neutral network, thus obtaining five groups of training samples. Data of the 7th day are deemed to be test samples of neutral network to test a prediction effect of neutral network.

First, the genetic algorithm is used for optimizing weights and thresholds of neutral network. The initial weight and threshold of neutral network are gained after 100 times of optimized iteration. The evolution curve of fitness function during a process of optimized iteration sees the figure 2.

From the figure 2, superposition between mean fitness and maximum fitness achieves 0.01762 after 60 times of genetic iteration, thus accomplishing search in overall situation, getting the initial weight and threshold of neutral network and confirming initial optimal solution. And then, the L-M algorithm is adopted to train the neutral network accurately in order to obtain optimal solution of weight and threshold, therefore, the neutral network training finishes.The convergence curve of neutral network training with L-M algorithm sees the figure 3. The convergence of neutral network training with BP algorithm sees the figure 4.

From comparison between figure 3 and figure 4, the L-M algorithm just needs 4 steps of mean square error to convergent to a magnitude of $10-6$ and achieve $1.04 \times 10-7$ with a rapid training speed as the genetic algorithm has founded initial optimal solution according to genetic optimization. On this basis, the L-M finishes the training rapidly in a rate of second-order convergence; however, the BP algorithm uses a gradient descent method, and has an error of $9.09 \times 10-4$ and still cannot achieve the training goal after 500-step training. So, the L-M algorithm is capable of effectively solving the problem that the BP algorithm has a slow training speed and long time, greatly improving the training efficiency. And meanwhile, the simulation result shows 
that the effect is more ideal to mix the genetic algorithm and LM neutral network.

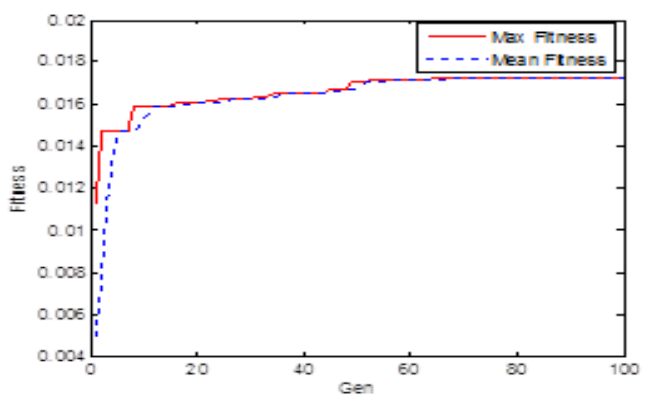

Fig.2. Evolution curve of fitness function

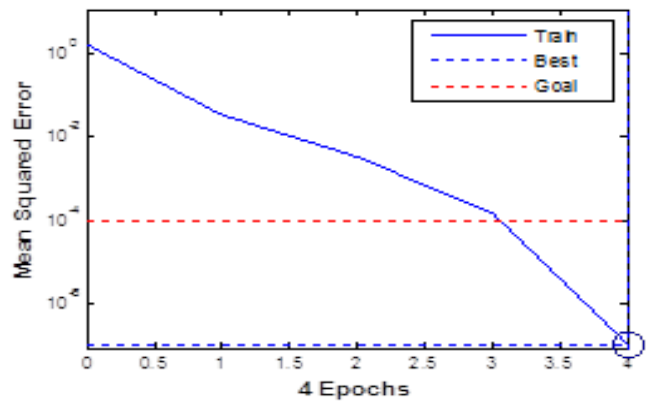

Fig.3. Convergence curve of neural network training with L-M algorithm

In order to further prove effectiveness of short-term load prediction of power system of genetic neutral network, the paper makes use of the genetic neutral network model which finished the training to predict the short-term load, and then compares it with actual load data. And at the same time, the curve of prediction load and actual load is drawn. In the figure, the red full line stands for an actual load change chart, the blue dotted line means a load change curve achieved by L-M algorithm prediction, and the green dotted line is a load change curve achieve by BP algorithm prediction, which see figure 5 .

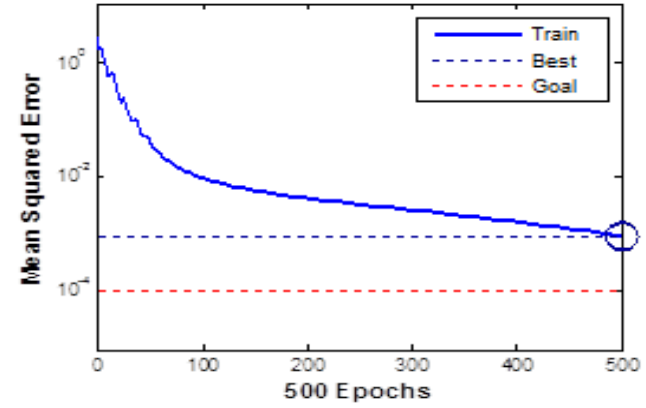

Fig.4. Convergence curve of neural network training with BP algorithm

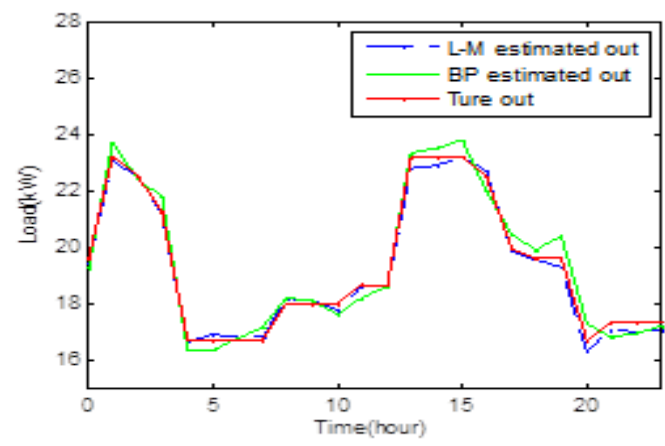

Fig.5. Curve of prediction load and actual load

From the figure 5, compared with the BP neutral network, the effect of short-term load prediction of power system, which uses the genetic neutral network of L-M algorithm, is more ideal. The table 1 lists prediction values and errors of genetic neutral network in detail.

Tab.1 Prediction load values and error on 5 April 2012

\begin{tabular}{cccc}
\hline Time $(\mathrm{h})$ & Actual value $(\mathrm{kW})$ & Predicted value $(\mathrm{kW})$ & Error $(\%)$ \\
\hline $0: 00$ & 19.63 & 19.43 & 1.02 \\
$1: 00$ & 23.17 & 23.12 & 0.23 \\
$2: 00$ & 22.52 & 22.46 & 0.27 \\
$3: 00$ & 21.24 & 21.18 & 0.27 \\
$4: 00$ & 16.73 & 16.67 & 0.34 \\
$5: 00$ & 16.73 & 16.91 & 1.05 \\
$6: 00$ & 16.73 & 16.82 & 0.52 \\
$7: 00$ & 16.73 & 16.82 & 0.53 \\
$8: 00$ & 18.02 & 18.16 & 0.77 \\
$9: 00$ & 18.02 & 18.09 & 0.40 \\
$10: 00$ & 18.02 & 17.78 & 1.34 \\
$11: 00$ & 18.66 & 18.60 & 0.30 \\
$12: 00$ & 18.66 & 18.63 & 0.15 \\
$13: 00$ & 23.17 & 22.80 & 1.61 \\
$14: 00$ & 23.17 & 22.88 & 1.24 \\
$15: 00$ & 23.17 & 23.20 & 0.13
\end{tabular}




$\begin{array}{llll}\text { 16:00 } & 22.52 & 22.71 & 0.83 \\ 17: 00 & 19.95 & 19.84 & 0.57 \\ \text { 18:00 } & 19.63 & 19.54 & 0.45 \\ \text { 19:00 } & 19.63 & 19.30 & 1.69 \\ \text { 20:00 } & 16.73 & 16.34 & 2.33 \\ 21: 00 & 17.37 & 17.08 & 1.68 \\ 22: 00 & 17.37 & 17.01 & 2.06 \\ \text { 23:00 } & 17.37 & 17.05 & 1.83\end{array}$

It can be seen from the table 1 that, the genetic neutral network model can effectively predict the short-term power system load. And the maximum prediction error is $2.33 \%$ and the minimum prediction error is $0.15 \%$, achieving a requirement of $5 \%$ for the short-term load. Because the paper takes full account of influences to the prediction model by factors like air temperature and holiday, hence, the prediction model become more reasonable and the prediction effect is more ideal. In order to further compare the prediction effect between L-M algorithm and BP algorithm, the table 2 gives prediction accuracy of L-M algorithm and BP algorithm.

Tab.2 Prediction accuracy of L-M algorithm and BP algorithm

\begin{tabular}{cc}
\hline Algorithm & Mean absolute error (\%) \\
\hline L-M Algorithm & 0.91 \\
BP Algorithm & 3.56 \\
\hline
\end{tabular}

We can see from the table 2, the error of neutral network which uses the L-M training algorithm decreases by $2.65 \%$ than that of BP neutral network. This because the L-M training algorithm combines Gauss-Newton method and gradient descent method, adopts approximate second derivative information, thus becoming faster than the gradient descent method and has a training effect which is obviously better than BP algorithm. Therefore, the training time can be effectively shortened, and the prediction error decreases and the prediction accuracy and generalization ability increase.

\section{References}

[1] Wu Wang, Yuanmin Zhang, Ziliang Cai. Neural network based genetic algorithm optimizing for short-term load forecasting[J]. Relay. 2008,36(9):39-42.

[2] Tao Ren, Haijun Ma, Ning Fu. The research about the forecasting of power system load based on the method of genetic neural network[J].2012,21:67-68.

[3] Min Han, Dayun Mu. LM algorithm in echo state network for chaotic time series prediction[J]. Control and Decision.2011,26(10):1469-1472.

[4] Fei Han, Jianhua Mo, Pan Gong. Incremental sheet NC forming springback prediction using genetic neural network[J]. Journal of Huazhong University of Science and Technology(Nature Science Edition). 2008, 36(1): 121-124.

[5] Yi Luo, Feng Liu, Xiangjie Liu. Short-term wind power prediction based onprincipal component analysis and genetic neural network[J]. Power System Protection and Control. 2012,40(23):47-53. 